\title{
Azithromycin novel drug delivery system for ocular application
}

\author{
Ritu Mehra Gilhotra, Kalpana Nagpal' ${ }^{1}$ Dina Nath Mishra ${ }^{1}$
}

School of Pharmacy, Suresh Gyan Vihar University, Jaipur-302 030, 'Department of Pharmaceutical Sciences, Guru Jambheshwar University of Science and Technology, Hisar-125 001, India.

\begin{abstract}
Background: Azithromycin (AZT) is a macrolide antibiotic derived from and similar in structure to erythromycin. Oral administration of AZT is effective for the treatment of trachoma; however, topical formulations are difficult to develop because of the drug's hydrophobicity. The aim of this study is to formulate a novel topical ophthalmic delivery system of AZT. Materials and Methods: In the present study, ocular inserts of AZT are prepared using alginate, carbopol, and hydroxypropyl methylcellulose (HPMC) to solve the said formulation problem of drug and to facilitate ocular bioavailability. Ocular inserts were prepared by film casting method and the prepared films were subjected to investigations for their physical and mechanical properties, swelling behaviors, ex vivo bioadhesion, and in vitro drug release. Ocular irritation of the developed formulation was also checked by hen's egg chorioallantoic membrane test for ocular irritation potential. Results: The physicochemical, bioadhesive, and swelling properties of films were found to vary significantly depending on the type of polymers used and their combinations. The alginate films exhibited greater bioadhesion and showed higher tensile strength and elasticity than the carbopol films. HPMC addition to the films significantly affected the properties of ocular inserts. Carbopol:HPMC (30:70)-based ocular inserts sustained drug release for longest span of 6 $\mathrm{h}$. The release profile of AZT showed that drug release was by both diffusion and swelling. The formulation was found to be practically nonirritant in ocular irritation studies. Conclusion: AZT can therefore be developed as an ocular insert delivery system for the treatment of ocular surface infections.
\end{abstract}

Key words: Alginate, Azithromycin, Carbopol, HPMC, Ocular insert

\section{INTRODUCTION}

A major problem being faced in ocular therapeutics is the attainment of an optimal concentration at the site of action. Poor bioavailability of drugs from ocular dosage forms is mainly due to the tear production, nonproductive absorption, transient residence time, and impermeability of corneal epithelium. In addition to this, drugs that are hydrophobic or unstable at the $\mathrm{pH}$ comfortable in eye cannot be formulated as eye drops. Because of limitations of bioavailability pertaining to ocular route, there are many potent drugs, which still need to be studied for their

\section{Address for correspondence:}

Mrs. Ritu Mehra Gilhotra

School of Pharmacy, Suresh Gyan Vihar University,

Jaipur-302 033, India. E-mail: ritugilhotra@yahoo.co.in

\begin{tabular}{|l|l|}
\hline \multicolumn{2}{|c|}{ Access this article online } \\
\hline Quick Response Code: & Website: \\
\hline & www.jpionline.org \\
\cline { 2 - 3 } & \\
\hline
\end{tabular}

therapeutic potential by topical ocular route. Novel drug delivery systems could be some effective means of exploring the potential of such drugs. ${ }^{[1-4]}$

Azithromycin (AZT) binds to the 50S subunit of the $70 \mathrm{~S}$ bacterial ribosome and inhibits RNA-dependent protein synthesis. Ribosomal binding causes the death of bacteria or inhibits their growth depending on the organism, its sensitivity to AZT, and the concentration of AZT attained in the infected tissue ${ }^{[5,6]}$ Laboratory studies of aqueous AZT solutions have shown bactericidal capability against Staphylococcus aureus, Pseudomonas aeruginosa, and respiratory pathogens. ${ }^{[7,8]}$ In ophthalmology, oral administration of AZT has been proven effective for the treatment of trachoma. The treatment of ocular surface infections with topical AZT is desirable in medicine because systemic exposure to the drug is limited. ${ }^{[9]}$ Major topical formulation problems arise from the fact that AZT is hydrophobic and sparingly soluble in water at neutral $\mathrm{pH} .{ }^{[5]}$ Therefore, there is a need to design a novel drug delivery system, which could deliver the drug topically. Ocular inserts are polymeric systems into which the drug is incorporated as a solution (hydrophilic drug) or dispersion (hydrophobic drug) and molded as solid or semi-solid sterile preparations, of appropriate size and shape, designed to be inserted behind the eyelid or held on the eye and 
to deliver drugs for topical or systemic effect. There has been a growing interest in using bioadhesive films in the ocular cul de sac for enhanced or prolonged localized drug delivery. The polymers chosen in the present study were the biopolymer sodium alginate and the synthetic polymer Carbopol 981 NF. Hydroxypropyl methylcellulose (HPMC) was used in conjunction to enhance film properties.

\section{MATERIALS AND METHODS}

\section{Materials}

AZT was generously provided by IPCA Laboratory, India; HPMC K14 M was obtained as gift samples from Torrent Pharmaceuticals Ltd., India. Alginate was a gift sample from Snap Natural and Alginate Products Limited, Ranipet, and Carbopol ${ }^{\circledR} 981$ was purchased from Loba Chemie Pvt. Ltd., Mumbai.

\section{Methods}

\section{Preparation of ocular inserts ${ }^{[10,11]}$}

Films were prepared containing different ratios of Carbopol or alginate to HPMC [Table 1]. Ratio of plasticizer 50\% weight per gram of total polymer weight was used (Formulation code F1-F8). A $2 \%$ w/v polymeric solution was prepared with water and combined with plasticizer, the solution was centrifuged for $20 \mathrm{~min}$, and then casted onto a Petri dish and dried in an oven at $35^{\circ} \mathrm{C}$ until dry. The film was then removed from the Petri dish and cut to the required size. The films were stored in a glass container maintained at $25^{\circ} \mathrm{C}$ until use. Films with air bubbles or other imperfections were discarded. In manufacturing films containing AZT, the drug was added to the polymeric solution before casting onto a Petri dish. Five polymeric inserts for each formulation/batch were fabricated. Dried inserts were then cut into oval-shaped inserts with the help of a sharp-edged die (13.2 $\mathrm{mm}$ in length and $5.4 \mathrm{~mm}$ in width). Ocular inserts of the abovementioned dimensions with an area of approximately $77 \mathrm{~mm}^{2}$ were cut from the main insert, producing approximately 77 inserts for each batch. Each ocular insert contained theoretically 2.4 $\mathrm{mg}$ of the drug calculated on the basis of standard paper weight surface area method. ${ }^{[10,12]}$

\section{Physicochemical evaluation \\ Thickness of Insert ${ }^{[10,11]}$}

Thickness of the inserts $(n=3)$ was measured using dead weight thickness gauge (Prolific). After initial settings, the foot was lifted with the help of the lifting lever fixed on the side of the dial gauge. Insert was placed on the anvil such that the area where the thickness is to be measured lies below the foot. Readings of the dial gauge were recorded after gentle lowering of foot.

\section{Weight variation test ${ }^{[10,13]}$}

Inserts from each batch were randomly selected and weighed individually on electronic balance (AND HR 2000). Mean weight of inserts $(n=20)$ of each formulation was recorded.

\section{Surface $p H$ determination ${ }^{[10,11]}$}

Inserts were left to swell for $5 \mathrm{~h}$ on agar plate prepared by dissolving $2 \%(\mathrm{w} / \mathrm{v})$ agar in warm simulated tear fluid (STF; sodium chloride: $0.670 \mathrm{~g}$, sodium bicarbonate: $0.200 \mathrm{~g}$, calcium chloride. $2 \mathrm{H}_{2} \mathrm{O}: 0.008 \mathrm{~g}$, and purified water q. s. $100 \mathrm{~g}$ ) of $\mathrm{pH}$ 7.4 under stirring and then pouring the solution into Petri dish till gelling at room temperature. After the time of soaking, the $\mathrm{pH}$ of the wet surface was measured by placing the electrode in contact with the surface of the insert.

\section{Drug content uniformity ${ }^{[10,14]}$}

Uniformity of the drug content was determined by assaying the individual inserts. Each insert was grounded in a glass pestle mortar and $5 \mathrm{~mL}$ of STF was added to make a suspension. The suspension so obtained was filtered and the filtrate was assayed spectrophotometrically at $215 \mathrm{~nm}$ (UV-Vis Systronics Spectrophotometer-106, Systronics India Ltd., India).

\section{Mechanical strength}

Ocular insert with good tensile strength and percent elongation would resist tearing due to stress generated by the blinking action of eye. The insert was cut into strips $(50 \times 10 \mathrm{~mm})$. Tensile strength and elongation at break was determined by modifying the method used by Mishra and Gilhotra. ${ }^{[1]}$ The apparatus consisted of a base plate with a pulley aligned on it. One aluminum clip was fixed on one end of the base plate, to which the insert $(n=3)$ was clipped. The other end of the insert was

\begin{tabular}{|c|c|c|c|c|c|c|}
\hline Formulation & $\begin{array}{c}\text { Alginate } \\
(\%)\end{array}$ & $\begin{array}{c}\text { Carbopol } \\
(\%)\end{array}$ & $\begin{array}{c}\text { HPMC } \\
(\%)\end{array}$ & $\begin{array}{c}\text { Thickness* } \\
\text { (mm) }\end{array}$ & $\begin{array}{l}\text { Weight }^{\#} \\
\text { (mg) }\end{array}$ & $\%$ Drug content \\
\hline $\mathrm{F}_{1}$ & 2.0 & - & - & $0.045 \pm 0.007$ & $7.50 \pm 0.21$ & $98.5 \pm 0.7$ \\
\hline $\mathrm{F}_{2}$ & 1.4 & - & 0.6 & $0.049 \pm 0.008$ & $7.10 \pm 0.70$ & $99.7 \pm 0.8$ \\
\hline $\mathrm{F}_{3}$ & 1.0 & - & 1.0 & $0.050 \pm 0.005$ & $7.00 \pm 0.44$ & $99.5 \pm 0.3$ \\
\hline $\mathrm{F}_{4}$ & 0.6 & - & 1.4 & $0.051 \pm 0.007$ & $7.40 \pm 0.45$ & $98.6 \pm 0.0$ \\
\hline $\mathrm{F}_{5}$ & - & 2.0 & - & $0.055 \pm 0.003$ & $6.97 \pm 0.44$ & $97.9 \pm 0.1$ \\
\hline $\mathrm{F}_{6}$ & - & 1.4 & 0.6 & $0.051 \pm 0.008$ & $7.10 \pm 0.31$ & $99.8 \pm 0.4$ \\
\hline $\mathrm{F}_{7}$ & - & 1.0 & 1.0 & $0.050 \pm 0.007$ & $7.00 \pm 0.70$ & $98.4 \pm 0.2$ \\
\hline $\mathrm{F}_{8}$ & - & 0.6 & 1.4 & $0.050 \pm 0.003$ & $7.30 \pm 0.22$ & $98.5 \pm 0.8$ \\
\hline
\end{tabular}

HPMC, hydroxypropyl methylcellulose; AZT, azithromycin. Drug (AZT) content was $1 \%$ and plastisizer (PG) was $50 \%$ of total polymer content in all formulations. *Values given as mean $\pm S D(n=3) ;$ \#values as mean $\pm S D(n=20)$ 
clipped to movable aluminum clip. A thread was tied to movable clip and passed over the pulley, to which a small pan was attached to hold weights. A small pointer was attached to the thread that travels over the scale affixed on the base plate. The weights were gradually added to the pan till the insert (that was affixed between two clips) was broken. The weight necessary to break the insert was noted as break force and the simultaneous distance traveled by the pointer on the scale indicated the elongation at break. The following parameters were calculated as per equations:

Tensile strength $\left(\mathrm{g} / \mathrm{mm}^{2}\right)=$ break force $(\mathrm{g}) /$ cross-sectional area of the sample $\left(\mathrm{mm}^{2}\right)$

Elongation at break $(\mathrm{E} / \mathrm{B})(\%)=$ increase in length at break point $(\mathrm{mm})\left(L_{\mathrm{s}}-L_{\mathrm{o}}\right)$ /original length $\left(L_{\mathrm{o}}\right)(\mathrm{mm}) \times 100$

\section{Ex vivo bioadhesive strength}

Freshly excised conjunctiva of an adult goat was used as a model membrane for the measurement of bioadhesive strength. Whole eye bulbus of an adult goat was obtained from a local slaughter house, the underlying skin was removed to obtain freshly excised conjunctiva. The preparation was placed in an aerated saline at $4^{\circ} \mathrm{C}$ and later washed with distilled water and isotonic phosphate buffer ( $\mathrm{pH} 7.4,37^{\circ} \mathrm{C} \pm 1^{\circ} \mathrm{C}$ ) before use. Bioadhesive strength of insert $(n=3)$ was measured on a modified 2-arm physical balance. ${ }^{[14,15]}$ The pan at the left arm of the balance was detached and to the lever of left arm, was hanged a vertical thread, which had a rubber stopper tied to its end, hanging downward. Insert to be tested was adhered to the downward facing side of the rubber stopper. Goat conjunctival membrane was tied onto the open mouth of a glass vial filled with isotonic phosphate buffer. Vial was fitted in the center of a glass beaker filled with STF $\left(\mathrm{pH} 7.4,37^{\circ} \mathrm{C} \pm 1^{\circ} \mathrm{C}\right)$. The apparatus was set such that the vial (conjunctival membrane tied on it, facing upward) lies exactly below the rubber stopper (insert tied on it, facing downward). The rubber stopper was lowered so as to make the insert come in contact with the membrane. After facilitating the contact between the two, weight was put on right limb of balance, (gram force) required to detach the insert from the conjunctival surface gave the measure of detachment stress, calculated by:

Detachment stress $\left(\right.$ dyne $\left./ \mathrm{cm}^{2}\right)=[\mathrm{m} \cdot \mathrm{g} / \mathrm{A}]$

Where, $m$ is the weight required for detachment of insert, $g$ the acceleration due to gravity considered as $980 \mathrm{~cm} / \mathrm{s}^{2}$ and $A$ the area of tissue exposed $\left(\mathrm{cm}^{2}\right)$.

\section{Swelling index of prepared ocular inserts ${ }^{[10,11]}$}

Swelling of the polymer depends on the concentration of the polymer, ionic strength, and the presence of water. To determine the swelling index of prepared ocular inserts $(n=3)$, initial weight of insert was taken, and then placed in agar gel plate $(2 \%$ w/v agar in STF, pH 7.4) and incubated at $37^{\circ} \mathrm{C} \pm 1{ }^{\circ} \mathrm{C}$. For 5 $\mathrm{h}$, insert was removed from plate after every $1 \mathrm{~h}$, surface water was removed with help of filter paper, and insert was reweighed. Percent hydration was calculated.
Hydration $\%$ or $(\mathrm{Sw}) \%=[\mathrm{wt}-\mathrm{wo} / \mathrm{wo}] \times 100$

$(\mathrm{Sw}) \%=$ equilibrium percent swelling, wt $=$ weight of swollen insert after time $\mathrm{t}$,

wo $=$ original weight of insert at zero time

\section{In vitro drug release study}

The bichambered donor-receiver compartment model, designed using commercial semi-permeable membrane of transparent and regenerated cellulose type (Sigma dialysis membrane), was used to carry out the in vitro drug release studies. ${ }^{[13,16]}$ Semi-permeable membrane was used to mimic in vivo conditions, such as corneal epithelial barrier. The insert $(n=3)$ was placed in the donor compartment, and $7 \mu \mathrm{L}$ of STF with $\mathrm{pH} 7.4$ was maintained at the same level throughout the study in the donor compartment to simulate tear volume. The entire surface of the membrane was in contact with the reservoir compartment that contained 25 mL of STF with $\mathrm{pH} 7.4$, which was stirred continuously using a magnetic stirrer at $20 \mathrm{rpm}$ to simulate blinking action. Drug release was determined by withdrawing a defined quantity of sample from the sampling port at periodic intervals, which was replaced with equal volume of phosphate buffer $\mathrm{pH}$ 7.4. The drug content was analyzed at $215 \mathrm{~nm}$ using STF pH 7.4 as blank on UV-Vis Systronics Spectrophotometer-106.

The release data were kinetically analyzed using different kinetic models (zero-order, first-order, and Higuchi diffusion model). In order to determine the release model that best describes the pattern of drug release, the in vitro release data were fitted to zeroorder, first-order, and diffusion controlled release mechanisms according to the simplified Higuchi model. The equations used were as follows:

Zero-order kinetic model: $C=C_{\mathrm{o}}-K_{\mathrm{o}} t$

First-order kinetic model: $\log C=\log C_{\mathrm{o}}-K / 2.303$

Higuchi diffusion model: $Q=2 C_{\mathrm{o}}(D / \pi)^{1 / 2}$

where, $C_{o}$ is the initial drug concentration; $C$ the drug concentration (released) at time $t ; T$ the time of release; $Q$ the amount of drug released/unit area; $K_{\mathrm{o}}$ the zero-order rate constant; $K$ the first-order rate constant; and $D$ the diffusion coefficient, and it was calculated according to the following equation:

$D=\left(\right.$ slope $\left./ 2 C_{\mathrm{o}}\right) 2 \pi$

\section{Ocular tolerance}

For the present study, modified hen's egg chorioallantoic membrane (HET-CAM) test as reported by Velpandian et al, ${ }^{[16]}$ was carried out. Briefly, fertilized hen's eggs were obtained from poultry farm. Three eggs for each formulation weighing between 50 and $60 \mathrm{~g}$ were selected and candled to discard the defective ones. These eggs were incubated in humidified incubator at a 
temperature of $37^{\circ} \mathrm{C} \pm 0.5^{\circ} \mathrm{C}$ for 3 days. The trays containing eggs were rotated manually in a gentle manner after every $12 \mathrm{~h}$. On day 3 , egg albumin $(3 \mathrm{~mL})$ was removed by using aseptic techniques from the pointed end of the egg. The hole was sealed by $70 \%$ alcohol-sterilized parafilm (American Can Company, USA) with the help of a heated spatula. The eggs were kept in the equatorial position for the development of chorioallantoic membrane (CAM) away from the shell. The eggs were candled on the fifth day of incubation and every day, thereafter, nonviable embryos were removed. On the tenth day, a window $(2 \times 2 \mathrm{~cm})$ was made on the equator of the eggs through which formulations $(0.5 \mathrm{~mL})$ were instilled. A placebo film $(0.6 \%$ carbopol and $1.4 \%$ HPMC) was used as a control as it is reported to be practically nonirritant. The scores were recorded according to the scoring schemes as shown in Table 2 and score obtained was given in Table 3.

\section{Statistical analysis}

The results of mechanical strength, bioadhesive strength, swelling, and in vitro release were subjected to statistical analysis, using one-way analysis of variance (ANOVA) followed by $t$ test; $P<0.05$ was considered to be statistically significant.

\section{RESULTS AND DISCUSSION}

The present investigation was undertaken with the objective of preparing a sustained release bioadhesive ocular insert of AZT using alginate, carbopol, and HPMC as the matrix former as well as bioadhesive polymers. Propylene glycol (PG) was employed as plasticizer in the preparation to get inserts/films with good elasticity. The film casting procedure followed to prepare formulations resulted in the preparation of uniform AZT-polymeric bioadhesive inserts. ${ }^{[17]}$ The drug was dissolved in the polymeric solutions prior to casting. The concentration of the polymers plays an important part in the preparation of the polymer matrix. The solution of the insert was kept at room temperature for $24 \mathrm{~h}$ to enhance interdiffusion of polymer particles. Upon drying, polymer solutions were converted into drug polymer inserts/films. Various research groups have studied the mechanism of film formation using polymer dispersions. The film formation occurs in 3 stages: (a) evaporation of the casting solvent and subsequent concentration of polymer particles, (b) deformation and coalescence of polymer particles, and (c) further fusion by interdiffusion of polymeric molecules of adjacent polymer particles. The physical state of the drug in the dried polymer is dependent on the solubility of the drug in the polymer. In the present case, the drug was dispersed in the polymeric solution. The success of film formation method is further evident from the fact that the prepared inserts/films were translucent, colorless, and smooth in texture, uniform in appearance, thickness, and weight and showed no visible crack or imperfection. As a general observation, carbopol films (without HPMC) were highly elastic and sticky. Each ocular insert had an area of approximately $77 \mathrm{~mm}^{2}$. The insert had a thickness varying from $0.045 \pm 0.007 \mathrm{~mm}$ to $0.055 \pm 0.003 \mathrm{~mm}$. The dimensions of first ever commercially available ocular insert, OCUSERT ${ }^{\circledR}$ system by ALZA Corporation, Palo Alto, California; the Pilo-20 system is $5.7 \times 13.4 \mathrm{~mm}$ on its axes and $0.3 \mathrm{~mm}$ thick; the Pilo-40 system is $5.5 \times 13 \mathrm{~mm}$ on its axes and $0.5 \mathrm{~mm}$ thick. The prepared formulations were rather thinner than the commercially available

\section{Table 2: Scoring chart for HET-CAM test}

\begin{tabular}{lcl}
\hline Effect & Scores & Inference \\
\hline No visible hemorrhage & 0 & Nonirritant \\
Just visible membrane discoloration & 1 & Mild irritant \\
Structures are covered partially due to membrane discoloration or hemorrhage & 2 & Moderately irritant \\
Structures are covered totally due to membrane discoloration or hemorrhage & 3 & Severely irritant \\
\hline
\end{tabular}

HET-CAM, modified hen's egg chorioallantoic membrane.

\begin{tabular}{|c|c|c|c|c|c|c|c|c|c|c|}
\hline \multirow{2}{*}{$\begin{array}{l}\text { Formulation } \\
\text { Placebo film as control }\end{array}$} & \multicolumn{10}{|c|}{ Scores } \\
\hline & $\begin{array}{l}\text { Time } \\
\text { (in min) }\end{array}$ & 0 & 5 & 15 & 30 & 60 & 120 & 240 & 480 & 1440 \\
\hline & Egg 1 & 0 & 0 & 0 & 0 & 0 & 0 & 0 & 0 & 0 \\
\hline & Egg 2 & 0 & 0 & 0 & 0 & 0 & 0 & 0 & 0 & 0 \\
\hline & Egg 3 & 0 & 0 & 0 & 0 & 0 & 0 & 0 & 0 & 0 \\
\hline & Mean & 0 & 0 & 0 & 0 & 0 & 0 & 0 & 0 & 0 \\
\hline \multirow[t]{4}{*}{ Developed formulation } & Egg 1 & 0 & 0 & 0 & 0 & 0 & 0 & 0 & 0 & 0 \\
\hline & Egg 2 & 0 & 0 & 0 & 0 & 0 & 0 & 0 & 0 & 0 \\
\hline & Egg 3 & 0 & 0 & 0 & 0 & 0 & 0 & 0 & 0 & 1 \\
\hline & Mean & 0 & 0 & 0 & 0 & 0 & 0 & 0 & 0 & 0.33 \\
\hline
\end{tabular}

HET-CAM, modified hen's egg chorioallantoic membrane 
ones, indicating their physiologic suitability. The weight of the prepared formulation varied from $6.97 \pm 0.44$ to $7.50 \pm 0.21 \mathrm{mg}$. The drug content was consistent in all batches and varied from $97.9 \% \pm 0.1 \%$ to $99.8 \% \pm 0.4 \%$ [Table 1$]$.

The strength and elasticity of the insert is reflected by the parameters, tensile strength (TS), and elongation at break $(E / B)$. A soft and weak insert is characterized by a low TS and $E / B$. A hard and brittle insert is defined by moderate TS, low $E / B$ value. A soft and tough insert is characterized by moderate TS, high $E / B$, whereas a hard and tough insert is characterized by high TS, $E / B$. Hence it is suggested that a suitable bioadhesive ocular insert should have a relatively moderate $T S, E / B$. In the present study, bioadhesive polymers cast from aqueous solvent were alginate, carbopol, and HPMC; Table 4 shows the mechanical properties of the prepared AZT ocular inserts. Addition of PG $(50 \% \mathrm{w} / \mathrm{w}$ of total polymer content) as a plasticizer gave the inserts good mechanical properties as evident from the satisfactory elongation at break parameters for all inserts. PG as a plasticizer allows the polymer-drug matrix molecules to move more freely resulting in an increase in the flexibility of the inserts. The tensile strength of the inserts was found between $0.42 \pm 0.03 \mathrm{~g} / \mathrm{mm}^{2}$ and $0.89 \pm 0.11$ $\mathrm{g} / \mathrm{mm}^{2}$ and $E / B$ ranged between $23.9 \% \pm 2.5 \%$ to $57.4 \% \pm 2.3 \%$. The alginate films showed the highest TS but the lowest $E / B$. Carbopol films had lower TS than alginate films and significantly higher elasticity than alginate film. Increasing HPMC content in alginate films enhanced TS as well as elasticity significantly. ${ }^{[18]}$ However, a similar effect was seen in carbopol films but was

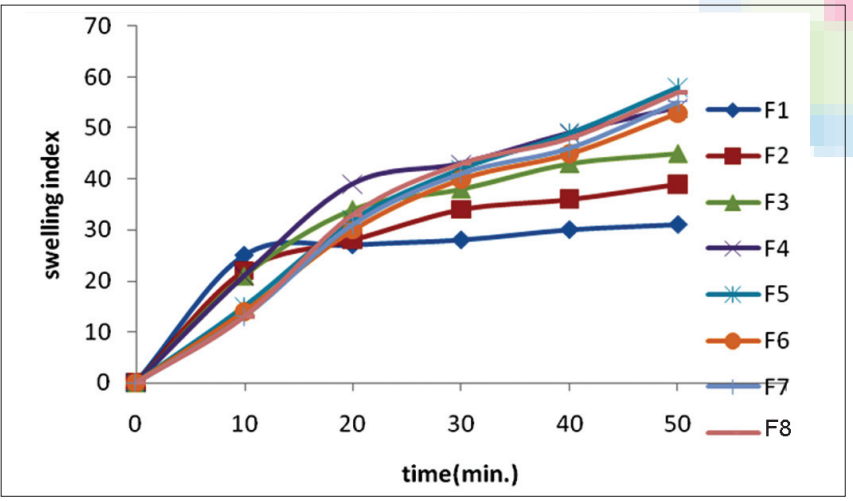

Figure 1: Swelling Index profile of prepared Azithromycin ocular Inserts insignificant. HPMC appeared to improve the properties of the alginate films by increasing tensile strength and elasticity. Formulation F1 showed a statistically significant difference $(P<0.05)$ in mechanical strength with respect to formulation $\mathrm{F} 4$ and formulation F5. Furthermore, formulation AF5 also showed a statistically significant difference $(P<0.05)$ in mechanical strength with respect to formulation F8.

Figure 1 shows the swelling profiles of prepared ocular inserts. Alginate films showed higher swelling or water uptake than carbopol film during initial study; ${ }^{[19-21]}$ however, the films reached equilibrium sooner. Increasing HPMC in alginate films enhanced the swelling of the films. This indicates the water retaining capacity of HPMC. Secondly, carbopol film had shown lower swelling than alginate film initially and the swelling of carbopol film did not appear to be affected by the presence of HPMC. Formulation F1 showed a statistically significant difference $(P<0.05)$ in swelling with respect to formulation $\mathrm{F} 4$. Furthermore, formulation AF5 also showed a statistically significant difference $(P<0.05)$ in swelling with respect to formulation F8.

The presence of HPMC in the films had a marked effect on the bioadhesiveness. For films without HPMC, the carbopol films showed the highest work of adhesion. For alginate films with higher HPMC, the work of adhesion was significantly higher $(P<0.05)$ than for films without HPMC. For carbopol films, the presence of HPMC caused a significant lowering $(P<0.05)$ of adhesive strength. In general the alginate films with HPMC showed higher bioadhesive strength than the carbopol films with HPMC.

The rate and the extent of insert hydration and swelling also affect the insert adhesion and consequently the drug release from the insert. Hence this parameter is important for predicting drug release as well as bioadhesive potential of matrix. Excessive hydration of a polymeric film could lead to decrease in adhesive strength and possibly loss of adhesion and hence a shorter duration of retention.

Figure 2 shows the in vitro release of AZT from polymeric inserts cast from aqueous solvent. It could be concluded from the results

\begin{tabular}{|c|c|c|c|c|}
\hline Formulation & $\begin{array}{l}\text { Tensile strength } \\
\left(\mathrm{g} / \mathrm{mm}^{2}\right)(\mathrm{n}=3)\end{array}$ & $\begin{array}{l}\text { Elongation at break } \\
(\%)(n=3)\end{array}$ & $\begin{array}{l}\text { Bioadhesive force } \\
(N)(n=3)\end{array}$ & $\begin{array}{l}\text { Detachment stress } \\
\left(\text { dynes } / \mathrm{cm}^{2}\right)(\mathrm{n}=3)\end{array}$ \\
\hline $\mathrm{F}_{1}$ & $0.79 \pm 0.05$ & $23.9 \pm 2.5$ & $7.5 \pm 0.21$ & 0.0735 \\
\hline $\mathrm{F}_{2}$ & $0.84 \pm 0.02$ & $29.3 \pm 4.5$ & $8.9 \pm 0.30$ & 0.0873 \\
\hline $\mathrm{F}_{3}$ & $0.89 \pm 0.11$ & $33.5 \pm 8.5$ & $9.8 \pm 0.71$ & 0.0961 \\
\hline $\mathrm{F}_{4}$ & $0.93 \pm 0.07$ & $38.2 \pm 4.5$ & $12.3 \pm 0.21$ & 0.1206 \\
\hline $\mathrm{F}_{5}$ & $0.42 \pm 0.03$ & $50.5 \pm 4.2$ & $18.9 \pm 1.20$ & 0.1854 \\
\hline $\mathrm{F}_{6}$ & $0.48 \pm 0.07$ & $52.3 \pm 1.5$ & $13.2 \pm 0.80$ & 0.1296 \\
\hline $\mathrm{F}_{7}$ & $0.53 \pm 0.02$ & $56.0 \pm 4.0$ & $11.0 \pm 0.11$ & 0.1079 \\
\hline $\mathrm{F}_{8}$ & $0.59 \pm 0.08$ & $57.4 \pm 2.3$ & $8.4 \pm 0.20$ & 0.0824 \\
\hline
\end{tabular}

\footnotetext{
AZT, azithromycin
} 


\begin{tabular}{|c|c|c|c|c|c|c|c|c|}
\hline \multirow{2}{*}{$\frac{\text { Formulation }}{\text { F8 }}$} & \multicolumn{2}{|c|}{ Zero order } & \multicolumn{2}{|c|}{ First order } & \multicolumn{2}{|c|}{ Higuchi diffusion } & \multicolumn{2}{|c|}{$\log Q V s / \log t$} \\
\hline & $\mathrm{R}$ & $\mathrm{K}$ & $\mathrm{R}$ & $\mathrm{K}$ & $\mathrm{R}$ & $\mathrm{K}$ & $\mathrm{R}$ & $\mathrm{n}$ \\
\hline & 0.926 & 0.041 & 0.976 & 2.7 & 0.978 & 0.235 & 0.956 & 0.73 \\
\hline
\end{tabular}

that the insert containing alginate (formulation code F1) gives the fastest release of the drug. It sustained the drug release for the shortest period of $3 \mathrm{~h}$. As stated in the swelling study, HPMC added to alginate films shows the potential of HPMC as a water retaining polymer, similarly adding HPMC content to alginate films enhances its potential of sustaining the drug release. Formulation F4 containing a higher HPMC content shows drug release up to $5 \mathrm{~h}$. This indicates that adding HPMC to alginate films helps sustain drug release. Carbopol films had shown slower drug release than alginate films. Also adding HPMC to carbopol had prolonged the drug release up to $6 \mathrm{~h}$. Formulation F8 having a higher content of HPMC sustained drug for the longest period of $6 \mathrm{~h}$. Statistical analysis (ANOVA) of the percent drug release showed that release of the drug from formulation F8 [Figure 2] was significantly lower than other formulations.

Formulation F8 was chosen as the optimized formulation on the basis of the longest span of drug release. Also, it has shown optimum physicochemical, mechanical, and bioadhesive parameters. Only formulation F8 was taken up for the kinetic modeling and ocular irritation studies. To determine the release mechanism that provides the best description to the pattern of drug release, the in vitro release data were fitted to zero-order, first-order, and diffusion-controlled release mechanism according to the simplified Higuchi model. The preference for a certain release mechanism was based on the correlation coefficient $(r)$ for the parameters studied, where the highest correlation coefficient is preferred for the selection of the mechanism of drug release. ${ }^{[22]}$ Successive evidence of the relative validity of diffusion and first order models was obtained by further analyzing the data using the following equation:

$M / M_{\infty}=\mathrm{K} . t^{n}$

Where, $M / \mathrm{M}_{\infty}$ is the fraction released of the drug at time $t, \mathrm{~K}$ is a constant incorporating structural and geometric characteristics and $n$ is the release exponent characteristic for the drug transport mechanism. When $n=0.5$, Fickian diffusion is observed and the release rate is dependent on $t$, whereas $0.5<n<1.0$ indicate anomalous (non-Fickian) transport and when $n=1$, the release is zero-order. The mathematical treatment of the in vitro release data of AZT from the optimized ocular insert (F8) is presented in Table 5. The optimized ocular insert F8 follows the firstorder kinetics. Values of $n=0.73$ of formulation supported an anomalous non-Fickian release. As known, $n=0.5$ (indicating diffusion-controlled drug release) and $n=1.0$ (indicating swelling-controlled drug release). Values of $n$ between 0.5 and 1.0 can be regarded as for the superposition of both the phenomena (anomalous transport). Hence, in the present case, the drug release from the matrix is controlled by both phenomena, that is, diffusion and swelling. ${ }^{[23,24]}$

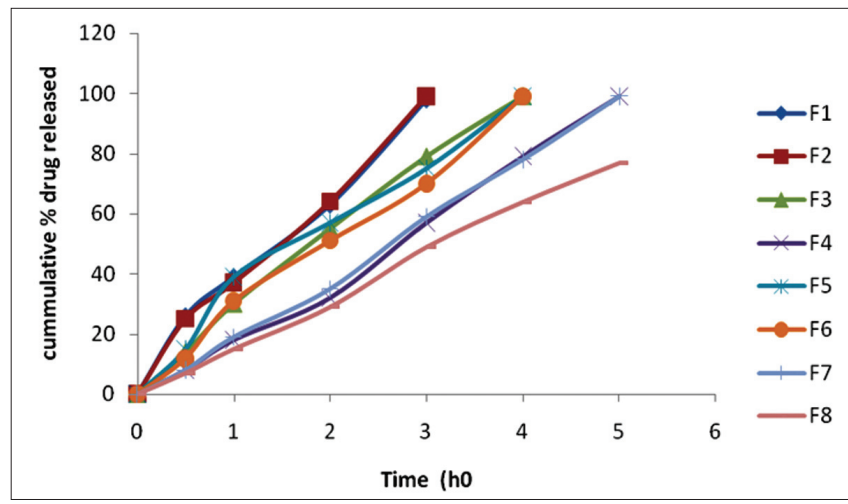

Figure 2: Drug release profile of prepared Azithromycin ocular films

The HET-CAM is a suitable alternative to animal testing (Draize test $)^{[25]}$ and it is based on the direct application onto the CAM of the sample and reactions, such as hemorrhage, intravasal coagulation, or lysis of blood vessels are microscopically assessed on a time-course basis. ${ }^{[26]}$ These irritancy effects may occur within 5 min following mucosal administration of sample, directly onto the CAM of a hen's egg, according to the Invittox protocol. ${ }^{[27,28]}$

The CAM is a noninnervated complete tissue containing arteries, veins and capillaries and it is technically easy to study. It responds to injury with an inflammatory process similar to what one would observe in the conjunctival tissue of a rabbit's eye. Its well-developed vascularization provides an ideal model for ocular irritation studies. Ocular irritation of the developed formulation was checked by hen's egg CAM test, which is a rapid, sensitive, and inexpensive test. Testing with incubated eggs is a borderline case between in vivo and in vitro systems and does not conflict with the ethical and legal obligations. The CAM of the chick embryo is a complete tissue, including veins, arteries, and capillaries, and is technically very easy to study. It responds to injury with a complete inflammatory process, a process similar to that induced in the conjunctival tissue of the rabbit eyes. The formulation developed was tested by this method and the result was compared with those obtained using normal saline, which was used as the control that is supposed to be practically nonirritant. A mean score of 0 was obtained for the placebo film. Carbopol/HPMC-based formulation was nonirritant up to 12 h (mean score 0 ), whereas the mean score was found to be 0.33 up to $24 \mathrm{~h}$ [Table 4]. The study shows that the formulation is nonirritant to mild irritant and is well tolerated.

\section{CONCLUSION}

Most of the currently marketed ocular drugs were initially developed for nonocular applications. Recently, tremendous research is carried out to make orally available drugs applicable 
for topical ocular therapeutically effective by the help of polymer system fabrication. AZT can be developed as an ocular insert delivery system for the treatment of ocular surface infections. The ocular delivery system of AZT based on carbopol and HPMC has shown a sustained drug release as well as good ocular tolerability. This dosage form is a good means of making the hydrophobic drug, which is topically delivered. The development of AZT in this delivery system could enhance the antibiotic's usefulness in ophthalmology for the topical treatment of ocular surface bacterial infections and lid margin diseases. Further work to check the therapeutic potential of the dosage form in vivo is in progress.

\section{REFERENCES}

1. Bourlais CL, Acar L, Zia H, Sado PA, Needham T, Leverge R. Ophthal drug del sys recent adv. Prog Retinal Eye Res 1998; 17:33-58.

2. Ding S. Recent developments in ophthalmic drug delivery. Pharm Sci Technol Today 1998;1:328-35.

3. Saettone MF, Salminen L. Ocular inserts for topical delivery. Adv Drug Del Rev 1995;16:94-106.

4. Mitra AK, editor. Ophthal Drug Del Sys. New York: Marcel Dekker; 1993. p. 261-75.

5. Sevillano D, Alou L, Aguilar A, Echevarría O, Giménez MJ, Prieto J. Azithromycin IV pharmacodynamic parameters predicting streptococcus pneumonia killing in epithelial lining fluid versus serum: An in vitro pharmacodynamic situation. J Antimicrob Chemother 2006;57:1128-33.

6. Retsema J, Fu W. Macrolides: Structures and microbial targets. Int J Antimicrob Agents 2001;18:3-10.

7. Meyer AP, Bril-Bazuin C, Mattie H, Broek VP. Uptake of azithromycin by human monocytes and enhanced intracellular antibacterial activity against Staphylococcus aureus. Antimicrob Agents Chemother 1993;37:2318-22.

8. Imanura $\mathrm{Y}$, Higashiyama $\mathrm{Y}$, Tomono K, Izumikawa K, Yanagihara $\mathrm{K}$, Ohno $\mathrm{H}$, et al. Azithromycin exhibits bactericidal effects on Psuedomonas aeruginosa through interaction with the outer membrane. Antimicrob Agents Chemother 2005;49:1377-80.

9. Bowman LM, Si E, Pang J, Archibald R, Friedlaender M. Development of a topical polymeric mucoadhesive ocular delivery system for azithromycin. J Ocular Pharmacol Therap 2009;25:2.

10. Gilhotra RM, Gilhotra N, Mishra DN. Piroxicam bioadhesive ocular inserts: Physicochemical characterization and evaluation in prostaglandin-induced inflammation. Curr Eye Res 2009;34:1065-73.

11. Mishra DN, Gilhotra RM. Design and characterization of bioadhesive in-situ gelling ocular inserts of gatifloxacin sesquihydrate. DARU 2008;16:1-8.

12. Sultana Y. Development and evaluation of ocular drug delivery systems. Ph.D. Thesis, submitted at Faculty of Pharmacy, Jamia Hamdard (Hamdard University), Hamdard Nagar, New Delhi, India: 2002.

13. Sreenivas SA, Hiremath SP and Godbole AM. Ofloxacin ocular inserts: Design, Formulation and Evaluation. Iranian J Pharmacol Therap 2006;5:159-62 .

14. Dandagi PM, Manvi FV, Patil MB, Mastiholimath VS, Rathod R. Development and evaluation of ocular films of cromolyn sodium. Indian J Pharm Sci 2004;66:309-12.

15. Sultana Y, Aqil M, Ali A, Zafar S. Evaluation of carbopol-methyl cellulose based sustained release ocular delivery system for pefloxacin mesylate using rabbit eye model. Pharm Dev Tech 2006;11:313-9.

16. Gorle AP, Gattani SG, Design and evaluation of polymeric ocular drug delivery system. Chem Pharm Bull (Tokyo) 2009;57:914-9.

17. Khanna R, Agarwal SP, Ahuja A. Preparation and evaluation of mucoadhesive buccal films of clotrimazole for oral Candida infections. Indian J Pharm Sci 1997;59:299-305.

18. Liu Z, Li J, Nie S, Liu H, Ding P, Pan W. Study of an alginate/ HPMC-based in situ gelling ophthalmic delivery system for gatifloxacin. Int J Pharm 2006;315:12-7.

19. Smidsrod O, Skjakbraek G. Alginate as immobilization matrix for cells. Trends Biotechnol 1990;8:71-8.

20. Kim CK, Lee EJ. The controlled release of blue dextran from alginate beads. Int J Pharm 1992;79:119.

21. Smadar C, Esther L, Amira T, Yale P. A novel in situ-forming ophthalmic drug delivery system from alginates undergoing gelation in the eye. J Control Rel 1997;44:201-8.

22. V'Ooteghem MM. Biopharmaceutics of Ocular Drug Del. In: Edman P, editor. Boca Raton: CRC Press; 1993. p. 27-41.

23. Charoo NA, Kohli K, Ali A, Anwer A. Ophthalmic delivery of ciprofloxacin hydrochloride from different polymer formulations: In vitro and in vivo studies. Drug Dev Indus Pharmacy 2003;29:215-21.

24. Schwartz JB, Simonelli AP, Higuchi WI. Drug release from wax matrices: Analysis of data with first order kinetics and with the diffusion controlled model. J Pharm Sci 1968;57:274-7.

25. Luepke N. Hen's egg chorioallantoic membrane test for irritation potential. Food Chem Toxic 1985;23:287-91.

26. Spielmann H, Liebsch M, Kalweit S, Moldenhauer F, Wirnsberger $\mathrm{T}$, Holzhutter $\mathrm{H}$. Results of a validation study in Germany on two in vitro alternatives to the Draize eye irritation test, HET-CAM test and the 3T3 NRU cytotoxicity test. ATLA 1996;24:741-858.

27. Gilleron L, Coecke S, Sysmans M, Hansen E, Oproy SV, Marzin $\mathrm{D}$, et al. Evaluation of a modified HET-CAM assay as a screening test for eye irritancy. Toxic In Vitro 1996;10:431-46.

28. Grant GT, Morris ER, Rees DA, Smith PJ, Thom D. Biological interactions between polysaccharides and divalent cations: The egg-box model. FEBS Lett 1973;32:195-8.

Source of Support: Nil, Conflict of Interest: None declared Received : 14-10-10, Revised: 17-11-10, Accepted: 18-11-10

\section{Dispatch and return notification by E-mail}

The journal now sends email notification to its members on dispatch of a print issue. The notification is sent to those members who have provided their email address to the association/journal office. The email alerts you about an outdated address and return of issue due to incomplete/incorrect address.

If you wish to receive such email notification, please send your email along with the membership number and full mailing address to the editorial office by email. 